\section{ALIMENTARY TOXAEMIA.}

Papers Read at The Opening of a Disclision at THE ROYAl SOCIETY OF MEDICINE.

\section{GENERAL INTRODUCTION.}

By W. HaLE WhiTE, M.D.LoNd. ANd Debl., PHYSICIAN TO GUY'S HOSPITAL.

THE term "alimentary toxaemia" at once shows our ignorance, for it is unscientific to group cases of poisoning according to the point of entrance of the poison; they should be grouped according to the variety of the poison; it is a minor matter whether our murderer comes in at the window or the door. Further, the term is too wide; an illness due to any poison absorbed anywhere from the mouth to the anus is strictly an alimentary toxaemia, but the phrase would hardly cover a case of opium poisoning. The user of it probably wishes to suggest that an illness is due to poisons made by micro-organisms resident in the alimentary canal, although, with our present knowledge; lie can never name the poisons, nor is he certain of the offending micro-organisms; still we are equally ignorant of the poison in uraemia and diabetic coma. But it is not clear that it may not be used when micro-organisms from the alimentary tract-for example, Bacillus coli-pass into the blood and so cause illness, and it is somewhat confusing that if infected food causes poisoning we talk of food poisoning, not food toxaemia ; on the other hand, when the food is not infected by pathogenic microorganisms but makes the person who eats it ill, he is by some said to be suffering from alimentary toxaemia-for example, as in a person who has urticaria after eating strawberries.

The simplest alimentary toxaemia is that due to pyorrhoea alveolaris. This local trouble in the month leads to imperfect mastication, hence large pieces of food are swallowed which cannot be digested, and if they contain micro-organisms which, if the mass were properly masticated, would be set free in the stomach and destroyed there, they may indirectly lead to the production of poisons in the intestine. Further, the micro-organisms discharged in the pus from the gums may be swallowed, and it is possible that the gastric secretions, being abnormal or deficient, may not kill them, or that if the stomach is well filled with solid food and the micro-organisms are then washed down with a draught of water, they, suspended in the water, will be rapidly passed through the stomach on the top of the solid food, and hence reach the intestine and cause intestinal toxaemia. It may be that in some diseases micro-organisms found in the intestine have been exereted from the blood, and that such organ. isms may when in the intestine manufacture poisons. Micro-organisms taken into the blood from the diseased gums may perhaps cause intestinal toxaemia in this way. Lastly, the micro-organisms in the gums may pass into the blood, and cause septicaemia varying from mild to serere. I have met with several fatal cases, and very many persons owe general ill-health or chronic indigestion to pyorrhoea. It is surprising how often this is overlooked. When seeing a patient for the first time, it is as important to examine the teeth as to feel the pulse. Although most common among poor, ill-educated people, pyorrhoea is often to be found in those who presumably clean their teeth properly and frequently; sufferers from it are unaware that they have anything amiss with their teeth. Its presence does not prevent the patient from having other illnesses; sometimes these are wrongly attributed to pyorrhoea. It is dangerous to remove too many affected teeth at once, for a rapid absorption of micro-organisms may take place by the damaged vessels. The value of vaccines will be discussed later by others. My own practice is to give them, for I have never known them do harm in this condition, and I think I have seen them do good.

When we consider bacterial poisons formed in the gastro-intestinal tract, the difficulties are so great that we may almost despair of understanding the subject. External temperature is said to play a part, for although in temperate and tropical climates the lower intestines of animals which have been weaned swarm with micro- organisms-a human being excretes daily 128 billions, 99 per cent. of which are dead, in the faeces-yet the intestinal contents of arctic animals are by some observers stated to be sterile, or almost so, suggesting that it would be a good thing to establish at Spitzbergen a health resort for those thought to be suffering from alimentary toxaemia. Ill understood alterations in the gastro-intestinal contents must influence their bacteriology; thus, Bacillus coli is usually confined to the large intestine, but I know a woman suffering from severe dyspepsia and in whom lavage always shows the contents of the stomach to be swarming with Bacillus coli. Then the presence of one micro-organism affects others; for example, one of the chief functions of the Bacillus coli is to smother others, either by making bodies poisonous to them or by withdrawing their nourishment; it thus kills typhoid bacilli-a fact of interest when we remember that Bacillus coli may often be found in the urine of those afflicted with typhoid fever. On the other hand, the Bacillus coli may itself be killed by invaders-for example, Morgan's bacillus, B. suipestifer, $B$. enteritidis. Then the same micro-organism may act differently in different hosts-for example, one person's strain of colon bacillus often differs from another's. Again, alterations at one part of the intestine will affect processes going on lower down; for instance, colon bacilli have no effect on proteins, but they decompose (putrefy) peptones. If, therefore, there is complete absorption in the small intestine no peptones will reach the large, and so there will be no putrefaction, nor will there be if only unaltered proteins reach the large intestine, but any peptones will putrefy. We hope to hear from the bacteriologists which are the micro-organisms causing alimentary toxaemia. Herter taught us that there are probably threo groups of cases :

(a) The indolic, in which the probable fault was that the colon bacillus invaded the lower part of the small intestine, and the patient was unable to digest carbohydrates, and usually passed abundance of indican.

(b) The saccharo-butyric, in which the organism mostly concerned is the $B$. aerogenes capsulatus; the abnormal changes here occur in the large intestine.

(c) A group combined of $a$ and $b$.

We must, when thinking of alimentary toxaemia, bear in mind that the culture medium itself is as important as the bacteria, and therefore alterations of diet may influence the production of intestinal poisons as completely as bacteria themselves. A good instance of this is the improrement that follows the withdrawal of carbohydrates in cases of carbohydrate dyspepsia.

Those who ascribe most to alimentary toxaemia are unable to tell us what the poisons are. Much work has been done on the excretion of indican and ethereal sulphates in the urine, and there is no doubt that an excess of indican is often associated with serious intestinal disturbance, but many persons pass large amounts of indican for many years and remain in good health; on the other hand, indican is not passed in many instances in which the patient might be thought to have alimentary toxaemia. It is generally allowed that the poison is not indol, indican, or ethereal sulphates. Indeed, just as we do not know what the poison is in uraemia or diabetic coma, so we do not know what it is in the illnesses ascribed to intestinal toxaemia, nor do we know for certain which are, in any case, the offending microorganisms, nor do we understand the circumstances farouring their appearance and development, and, unfortunately, the circumstances cannot be exactly reproduced in the laboratory. Further, it would not necessarily follow that if, say, by subcutaneous injection certain symptoms were produced, the same would follow when the poison was absorbed from the intestine; mercury inhaled causes tremors, but not when taken by the mouth. Surely all this being so, statements should be made cautiously, yet one reads that indol-forming organisms must be harmful because they are prominent, and many equally foolish arguments. Nevertheless, some very suggestive work has been recently done by Mellanby and Twort and others, who have isolated creatin-destroying micro-organisms from the alimentary canal, and their experiments show that in the intestines of animals another bacillus forms $\beta$ imidazolethylamine, which is a powerful poison, from histidine, but the process cannot take place in an acid medium. 'This poison is probably destroved in the liver, 
and it is suggested may be a cause of cyclic vomiting. It would be very interesting to see if it is present in a piece of gut excised at an operation on the human subject. Bertrand and Berthelot have called the micro-organism B. aminophilus intestinalis.

But even if we knew all about the formation of the toxin, and had identified it in the intestine, our difliculties would not be at an end, for before it reached the general circulation it might be altered in its passage through the intestinal wall or in the liver. Indeed, it is possible that sometimes alimentary toxaemia may be a hepatic disease, in which substances absorbed from the intestine in all human beings are so altered in the liver of some that they become poisonous, or abnormal bodies arriving at the liver may so alter it that it produces poisons. Anylow, there is strong evidence that an important function of the liver is to protect us from poisons absorbed from the intestines, and that its efficient performance of this function depends upon carbohydrates. The difficulty is well illustrated by cirrhosis of the liver, which was formerly always ascribed to alcohol; but now we know that it oceurs in some who do not take alcohol, it does not occur in some who do, nor in animals poisoned by alcohol, and it is a part of some diseases in which alcohol plays no part-clearly. some other substances than alcohol can set up the cirrhotic process; and probably, under circumstances of which we know nothing, alcohol can accelerate the formation of these sub stances, which may well come from the intestine, and, if so, cirrhosis of the liver may be a form of alimentary toxaemia.

I am afraid our knowledge is not sufficient for us to argue that because certain processes go on in the intestine there must be a clinical condition to which the term "intestinal toxaemia." may be applied; but it does not follow that there are not assemblages of symptoms of which an alimentary toxaemia is such a likely cause that we may with reason assign them to this cause until some other cause is shown to exist. The best example of such an assemblage is that known as carbohydrate dyspepsia. An infant afflicted with this has pyrexia ; it loses flesh, but its tissues gain in water; its abdomen is distended by the accumulation of gas in the bowel; it is pale, fretful, and sleeps badly; it is often sick and has diarrhoea. The stools contain green colouring matter and an excess of fatty acids; they irritate the skin round the anus, leading to dermatitis. These symptoms may be produced in a previously healthy child if an excess of carbohydrate is given, and will pass away if that excess is withheld. I would suggest that these are the cases of intestinal toxaemia that should be first studied, for in them we see the problem at its simplest, the illness being induced by a single simple article of food. As this is carbohydrate and the intestines are distended with gas, there is every probability that excessive fermentation accompanies the formation of some poison which is capable of causing pyrexia; indeed, much older children often suffer from attacks of pyrexia which may be prevented by abstention from carbohydrates. Enterogenous cyanosis is a form of intestinal toxaemia from which we might learn much if it were commoner, for we know that in the methaemoglobinaemia variety there is a nitrite in the blood probably cerived from some abnormal intestinal process, and that in the sulph-haemoglobinaemia variety hydrogen sulphide is concerned with the production of the disorder, and as the cyanosis has disappeared when the accompanying constipation has been overcome there is every probability that the complaint is really an example of alimentary toxaemia. As nitrites and hydrogen sulphide are simple chemical bodies, it is particularly disappointing that we can discover nothing about the abnormal intestinal processes at the bottom of enterogenous cyanosis, and this should make us particularly careful of dogmatizing when we do not even know what the poisons are. Certainly sufferers from enterogenous cyanosis have not the symptoms which it is the fashion now to assign to alimentary toxaemia.

Lately it has been urged with much insistence that many suffer from ill health due to absorption of poisons from the bowel which are formed there because the bowel is imperfectly emptied ; or, in the modern terminology, these patients are said to have intestinal toxaemia due to intestinal stasis. That such is the case is very likely. Most people feel miserable if their bowels are not open for some time. Advertisements of medicines designed to keep the bowels open assail us all over the world many health resorts flourish exceedingly because their natural waters are aperient, colossal fortunes have been made out of the sale of purgatives, the Pharmacopoeia contains more examples of the aperient than of any other class of drugs, and the improvement in health that follows keeping the bowels properly open is often so striking that, from the clinical point of view, there is strong evidence that we may suppose such an alimentary toxaemia secondary to intestinal stasis, although we are unable to indicate the poison or to say how it is formed. But it must never be forgotten that individuals vary exceedingly about their bowels; some are perfectly well if the bowels are only open once a week. Presumably, if intestinal stasis is an important factor in causing alimentary toxaemia, these persons do not readily form poisons, or, if they do, they are immune to them. It has, however, been stated recently that the results of chronic alimentary toxaemia are much more numerous than was previously thought, and that the carse of the failure to empty the bowel is frequently mechanical and has been often overlooked.

To take the last point first. It is not necessary for the production of alimentary toxaemia that there should be any mechanical obstruction to the passage of the contents of the bowel. If the poisons are bacterial the quantity of them produced will depend upon the number of micro organisms and the suitability of the medium for their growth, which, for example, might be so favourable that toxaemia would result without any stasis; therefore toxaemia is not proof of stasis: Those who attach great importance to mechanical stasis differ much among them selves as to its cause. Some attribute it to excessively large valves of Houston, some to pericolitis, some to dropping of the colon, some to adhesions around the ascending colon, some to spur formations at the hepatic and splenic flexures, some to abnormal mobility of the caecum, although others say that the mobility of the caecum thought to be abnormal is normal, and some to the development of various .bands and abnormal mesenteries that drag the bowel and kink it; but the reasons given for thinking that these bands are evolutionary are by no means convincing, most observers regard them as in flammatory. We have no sufficiently large number of cases proving that any of these conditions are more common in those supposed to be suffering from intestinal toxaemia than in those who are not, and it is strange that when tho bowels are by chronic peritonitis matted into one mass, so that there must be many abnormal kinks and great interference to peristalsis must exist, yet the symptoms said to be indicative of alimentary toxaemia are not constantly present, and even intestinal obstruction is rare. Although it may be that some of these mechanical causes lead to alimentary toxaemia, the matter cannot be regarded as proved, and it must be remembered that the coexistence of two phenomena does not prove that they stand in the relation of cause and effect; they may have a common cause-indeed, it is possible that the abnormal bands and the toxaemia are induced by the same cause. Much investigation with $x$ rays has been performed upon those thought to be suffering from alimentary toxaemia and there is no doubt that the $x$ rays have added and will add enormously to our knowledge of intestinal movements, both normal and abnormal, but we must bear in mind that although $x$ rays mày show a kink, they cannot show the cause of the kink; that if large amounts of such a heary substance as bismuth are used, the bowels can hardly be said to be acting normally; that we ought to bo provided with dozens of $x$-ray pictures of dozens of healthy people taking the same amount of bismuth; that when we look upon a screen we only see a plane picture. All inferences from $x$-ray examinations as to whether the duodenum or any other part of the bowel is dilated must be received with caution, for the rays from the $x$-ray tubes are divergent; we are dealing with a plane picture, and usually the statement is made that the duodenum, for example, is dilated without stating its size or that of the normal duodenum.

We may now turn to the symptoms which these sufferers from alimentary toxaemia are said to show. There is no need to detail them, for they have been recently described over and over again in the medical press. I am one of those who think it probable that they 
are due to alimentary toxaemia, but the poison has never been found, the symptoms have never been produced experimentally, few observers have up to the present even given an opinion on the matter, and in their extreme degree these symptoms are in my experience decidedly rare, yet we find it definitely stated, time after time, that these symptoms are due to alimentary toxaemia, but I submit that the evidence does not amount to proof, although it renders the suggestion probable. If we are not careful we shall, before we know where we are, be invoking the aid of alimentary toxaemia in the same reprehensibly loose way as gout, uric acid diathesis, and congestion of the liver have been called in to explain various vague symptoms which we do not understand.

So many conditions which cannot be called symptoms have been ascribed to intestinal stasis and consequent alimentary toxaemia, that they cannot all be examined now, but every one of them needs more careful scrutiny than it has received. Cystic disease of the breast, cancer of many parts, and duodenal ulcer, for example, have been set down to intestinal stasis, but it has not been shown that, among a large number of sufferers from any one of these, intestinal stasis is more common than among those in whom these conditions are not present. Then, intestinal stasis is commoner in women, duodenal ulcer in men, yet the first is said to cause the second. This casts so much doubt on the correctness of this suggested etiology that if there are special circumstances which can dispel the doubt they ought to be so convincingly stated that their adequacy is accepted by all. Nor have any of these conditions been induced in animals by artificially induced intestinal stasis. 'Then, again, we read that the "so-called Bright's disease is merely a product of intestinal stasis." There are many varieties of Bright's disease-which is meant? That being settled, let it be shown that a considerable proportion of those suffering from that variety of Bright's disease have alimentary toxaemia. This has not yet been done. Again, I see it suggested that curing alimentary toxaemia cured a case of exophthalmic goitre ; no such conclusion can be drawn from a single case, for exophthalmic goitre is a disease which tends to get well, especially if the patient rests, and this patient rested in bed during her operation for intestinal stasis. A woman suffering from exophthalmic goitre was admitted under me with typhoid fever; when she was discharged her exophthalmic goitre was cured. This does not show any relationship between typhoid fever and exophthalmic goitre, which was probably cured by the rest in bed.

If it is allowed that ill health is due to alimentary toxaemia caused by bacterial changes, the best treatment would be to stop the ingestion of the offending bacteria, but as we do not know what they are we cannot do this; or to give intestinal antiseptics, which experience has shown to be of limited use, although I think their uselessness has been exaggerated, for occasionally cyllin scems to do good; or to alter the medium in which the bacteria grow, which is successful when wo cure children by diminishing their carbolyydrates ; or to give other microorganisms which will render difficult the growth of the offending micro-organism, this is the principle of the sour milk cure, from which much was hoped but which has been disappointing; or if there is delay in emptying the intestine, to overcome this.

The last is the commonest mode of treatment, and thousands of people keep themselves in good health by natural aperient waters or some other simple aperienteither food or drug. Abdominal exercises and massage will, if properly carried out, cure many a case of constipa. tion which has resisted all other methods, and the same may be said of a visit to a liydropathic institution or a spa which lays itself out for the treatment of this disorder. Lately some surgeons have been trying to limit the formation of poisons in the intestines by preventing delay by periorming an ileo-sigmoidostomy either with or without excision of the colon. When these cases are reported we are always assured that all medical means had been adopted without benefit, but we are never told what the medical means were. It would add greatly to our knowledge if our surgical friends would give precise details of the medical treatment, the patient's statements being corroborated by the various doctors who treated her. A suspicion comes into one's mind sometimes that perhaps some surgeons do not know all the means the physician has at his command for the treatment of delayed action of the bowels.

Some have opened the appendix and washed the bowel through from the appendix to the anus; if the stasis is in the small intestine this operation will not be of much use, but the success which may follow it when performed for colitis is so brilliant that when the stasis is in the large bowel much benefit might be expected. One thing is certain-namely, that if surgical interference is ever necessary the patient for whom it is necessary is a reproach to her doctors if she has consulted any in the early periods of her illness, for the condition nerer ought to be allowed to advance to the stage which requires surgery, and, although no other operations may be feasible, yet short-circuiting and opening the appendix are only makeshifts, for neither removes the cause of the intestinal stasis.

It is too early to speak definitely as to the results of these short-circuiting operations. Occasionally the operation itself is fatal. I, like some others, have seen some women who are not in the least better for having had it done; on the other hand, many successes have been reported, but sometimes more than one operation has been required, and sometimes aperients are still necessary after operation. It would be a good thing if some of these cases were more fully reported; photographs before and a year after the operation would be useful, and the patients who had been operated on, whether successfully or not, might be shown before this society; and it is desirwble to have the after-history of every case that has ever undergone operation, for it may be that any improvemert that ensues is not permanent; this is especially important, as many of the subjects of operation are neurotic women, and we all know that the immediate effects of operation on them may be extraordinary. Further, it is unfortunate that the most frequent symptom-namely, pain-is subjective.

In this brief survey I have tried to indicate the direction which future work should take. I hope I have not been too critical; if any think so, I would beg them to remember that friendly criticism can do no harm. Our interest in alimentary toxaemia has been awakened by the fascinating suggestions of Metchnikoff and by the bold extension of them by Mr. Arbuthnot Lane, but it is an approved principle of science that the results of one worker cannot be accepted until confirmed by others. We ought, therefore, to withhold our verdict on many points until we see whether confirmation is forthcoming. In the year 600 A.D. Isidorus Hispalensis, Bishop of Seville, wrote: "For a medical man should know the ars rhetorica that he may be able to support with sound arguments the matters which he deals with; and also the ars dialectica, so that by the exercise of reason he may investigate the causes of sickness for the purposes of cure." That is true now as then.

\section{THE BACTERIOLOGY OF THE ALIMENTARY} CANAL.

BY F. W. ANDREWES, M.D., F.R.C.P., PATHOLOGIST AND LECTERER ON PATHOLOGY, ST, BARTHOLOMEN'S HOSPITAL, LONDON.

ThE interests of this discussion will, I think, best be served if I devote my allotted time first to a general review of the bacterial flora of the alimentary canal and then to a consideration of the part played by bacteria in the production of alimentary toxaemia. I must leave to others the more contentious details of this large subject.

\section{The Conditions for Bacterial Growth offered BY} the Alimentary Canal.

These conditions are for the most part ideal. Bacteria find there an equable temperature, and the moisture they require, while the supply of foodstuffs is ample and varied. Free oxygen is largely absent in the intestine, except perhaps in certain diarrhoeal conditions; we therefore find that the habitual tenants of the gut are facultative anaërobes, and that even strict anaërobes can grow there 